\title{
Evaluation of Effect of Moderate to Severe Chronic Periodontitis on Pulp of Teeth: A Histopathological Study
}

\author{
${ }^{1}$ Rithesh Kulal, ${ }^{2}$ Poorna Prakash, ${ }^{3}$ Mysore S Nalini, ${ }^{4}$ Remya Nath
}

\begin{abstract}
Introduction: The periodontitis affected teeth and the observed changes in the dental pulp have been controversial area of debate. The reason being the close communication between the periodontium and the pulp through the apical foramina and accessory lateral canals postulating the spread of infection between the tissue areas.
\end{abstract}

Aim: Evaluation of the histopathologic changes in dental pulp of teeth with moderate and severe chronic periodontitis.

Materials and methods: Human dental pulp was obtained using vital pulpectomy technique from 20 permanent teeth extracted due to hopeless periodontal prognosis. Pulp tissue samples were processed for histological examination and grading of changes observed.

Result: Inflammation was usual finding in the samples; however, only $8 \%$ of the teeth showed no inflammation. Pulp with moderate fibrosis was seen commonly in many sections. About $44 \%$ of samples showed edematous pulp.

Conclusion: Periodontitis can induce changes in the dental pulp considerations have to be given to the involved teeth for the depth of pocket and severity of periodontal involvement for proper treatment planning and management.

Keywords: Dental pulp, Histopathologic changes, Inflammation, Periodontitis.

How to cite this article: Kulal R, Prakash P, Nalini MS, Nath R. Evaluation of Effect of Moderate to Severe Chronic Periodontitis on Pulp of Teeth: A Histopathological Study. J Health Sci Res 2018;9(2):35-37.

Source of support: Nil

Conflict of interest: None

\section{INTRODUCTION}

Chronic periodontitis is chronic bacterial infection that affect the marginal periodontium, resulting in an inflammatory process that progressively alters the supporting

\footnotetext{
${ }^{1}$ Professor, ${ }^{2}$ Private Practitioner, ${ }^{3}$ Reader, ${ }^{4}$ Postgraduate Student

${ }^{1}$ Department of Periodontology, RajaRajeswari Dental College and Hospital, Bengaluru, Karnataka, India

${ }^{2-4}$ Department of Periodontology, Mysore Road Kumbalgodu, Bengaluru, Karnataka, India
}

Corresponding Author: Rithesh Kulal, Professor, Department of Periodontology, Rajarajeswari Dental College and Hospital Bengaluru, Karnataka, India, e-mail: rithesh14@yahoo.com and anchoring mechanisms of the teeth within the alveolar socket. ${ }^{1}$

The alterations observed in periodontium due to pulpal involvement are well documented; however, the influence of periodontal disease on the pulp of teeth has been debated. ${ }^{2}$ Studies in human beings and experimental animal models have shown that pulpal pathosis can cause varying degrees of periodontal changes. ${ }^{3}$ Following successful endodontic treatment, pathologic changes of endodontic origin usually disappear, and the periodontium returns to normal. ${ }^{2,3}$ Coyler postulated that actual and the inflammatory products of periodontitis could gain access to the pulp via accessory canals, apical foramina and dentinal tubules. ${ }^{4,5}$

\section{AIM}

This study was designed to evaluate and grade the possible histopathologic changes in the pulp of teeth with moderate and severe chronic periodontitis.

\section{MATERIALS AND METHODS}

The study was performed on human pulp tissue obtained from extracted teeth. The teeth included in the study had advanced periodontitis and questionable or hopeless prognosis and had at least one of these criteria:

- Radiographic evidence of advanced alveolar bone loss to $2 / 3$ rd of root length

- Grade II or III furcation involvement

- Grade III mobility.

The teeth were obtained from the Department of Oral and Maxillofacial Surgery of the RajaRajeswari Dental College and Hospital. Before extraction, oral examinations were done and radiographs were taken and only teeth with intact crowns and no restorations were selected. The teeth were extracted as atraumatically as possible under complete local anesthesia. Immediately following extraction, the histological samples were obtained from dentalpulpof the extracted teeth using the technique of vitalpulpectomy. The samples obtained were washed with a saline solution and fixed immediately in $10 \%$ formalin solution. After incubation for at least 7 days, the teeth were decalcified in $10 \%$ nitric acid for $10-15$ days. The specimens were histologically processed, embedded in paraffin, and serial sections of $5 \mu$ in thickness were tested with hematoxylin and eosin stains. 
Microscopic examination of each section was made. Each specimen of the pulp was assessed for degree of inflammation, amount of fibrosis, presence of pulp stones, presence of edema, and condition of pulpal vessels. The relative degree of inflammation was graded as follows: no inflammation (0-2 infiltrating cells); light inflammation (2-5 infiltrating cells); moderate inflammation (5-10 infiltrating cells); or severe inflammation (>10 infiltrating cells) (Figs 1 and 2). Fibrosis was defined as an increased fibroblast and collagen fiber concentration (Fig. 3). The relative degree of fibrosis was graded as follows: light fibrosis (3-10 fibroblast cells); moderate fibrosis (11-30 fibroblast cells); or severe fibrosis $(\geq 31$ fibroblast cells). The vessels were categorized into three groups (normal, atrophied, or dilated), according to their size. Edema was defined as accumulation of interstitial fluids in pulp.

\section{RESULTS}

Histological examination on pulp obtained from the teeth extracted for periodontitis showed variety of histological aspects that correlate with the severity of periodontal lesions. It was observed that inflammation was a seen in all the samples, with only $13.3 \%$ of teeth showing no inflammation. Inflammation observed in the sections was mostly moderate to severe and chronic (Figs 1 and 2). Forty percent of the sections showed fibrosis (Fig. 3), with only $5 \%$ of samples showing no fibrosis. Most teeth displayed edematous pulps (73.3\%). Pulp stones were absent in $86 \%$ of teeth. Finally, blood vessels showed congestive changes or discontinuities of the vascular wall, allowing extravasation of red blood cells (30\%), atrophic changes or intact vessels were also seen $(16.66 \%$ and 53.3\%) (Table 1).

Table 1: Histologic grading of the pulp of teeth with chronic periodontitis under various parameters

\begin{tabular}{llll}
\hline \multirow{2}{*}{ Parameter } & Grading & $\begin{array}{l}\text { Area/ } 60 \\
\text { fields }\end{array}$ & $\begin{array}{l}\text { Percentage } \\
(\%)\end{array}$ \\
\hline Inflammatory & No & 8 & 13.33 \\
cells & light & 26 & 43.3 \\
& moderate & 20 & 33.3 \\
Fibrosis & severe & 6 & 10 \\
& No & 3 & 5 \\
& slight & 16 & 26.6 \\
Blood vessels & moderate & 24 & 40 \\
& severe & 17 & 28.33 \\
& Dilated & 18 & 30 \\
Edema & normal & 32 & 53.3 \\
& atrophied & 10 & 16.66 \\
Calcification & Edema & 44 & 73.33 \\
& no edema & 16 & 26.66 \\
& Calcification & 8 & 13.33 \\
& no calcification & 52 & 86.66 \\
\hline
\end{tabular}

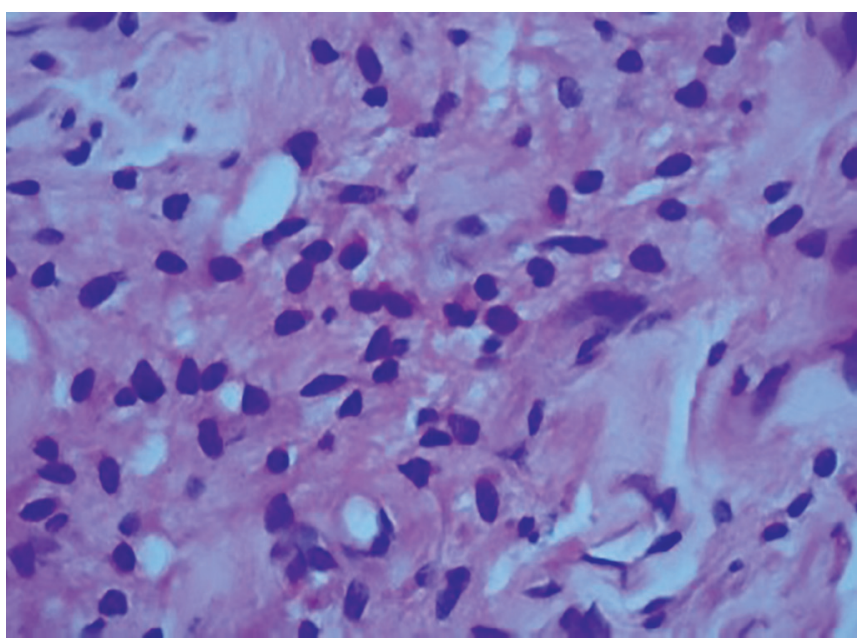

Fig. 1: Severe inflammatory cells ( $\mathrm{H}$ and $\mathrm{E} 40 \mathrm{X})$

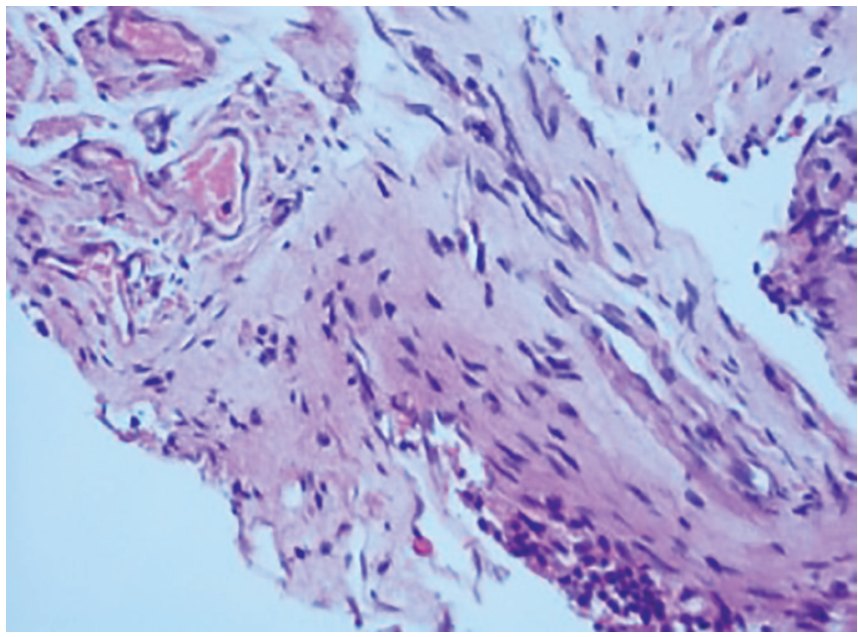

Fig. 2: Severe inflammatory cells seen with dilated blood vessels ( $\mathrm{H}$ and $\mathrm{E}$ 10X)

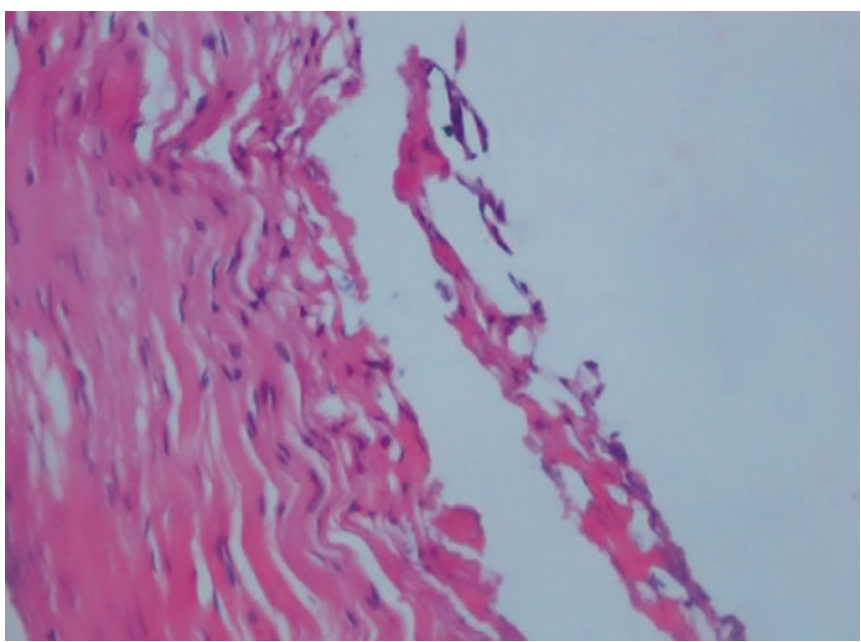

Fig. 3: Severe fibrosis seen (H and E $10 \mathrm{X})$

\section{DISCUSSION}

Prevalence of periodontal disease in individuals over 35 years is $50 \%$ and $30 \%$ of the teeth extracted are due to periodontal disease. ${ }^{6}$ This study was designed to evaluate the histopathologic changes associated with the dental pulp of teeth with chronic periodontitis. 
The anatomic and circulatory interrelationships have been the key evidence for the effect of periodontal disease on the pulp. ${ }^{2}$ On examination of the histologic sections of the pulpal tissue obtained from the study group exhibited clear effect of periodontal disease on the pulpal pathosis. Inflammation, fibrosis, edema, alteration in blood vessel and necrosis were considered as pulpal pathosis. Perfusion techniques have been used to study the vascular architecture of the human dental pulp and study observed the intricate relationship of pulpal and periodontal tissue. ${ }^{7,8}$ Teeth affected with periodontal disease have shown inflammatory lesions in both the apical and radicular areas of the pulp and this is could be attributed to the communication through lateral canal with the periodontal lesion resulting in secondary pulpitis. ${ }^{2,3}$

No pulp stones were observed in most of the teeth, similar to other studies. Rubach and Mitchell in their study mentioned that denticles, ranging from diffuse to concentric laminated bodies, were most common in coronal pulp. ${ }^{9}$

The noxious products from the microorganisms present in the periodontal lesions may injure the pulp cells. ${ }^{3,10}$ Moreover, the access of microorganisms through the lateral canals may describe the persistence of positive cultures in endodontic procedures. ${ }^{10}$ Significant variation in diameter and dilated pulpal vessels were detected in most of the teeth. Even with the presence of vessel dilatation, nonedematous pulp was seen in most teeth. Similar results were observed in one of the previous study of periodontally diseased teeth where all pulpal sections exhibited a generalized infiltration of inflammatory cells, comprising of lymphocytes, plasma cells, and macrophages with a few polymorphonuclear leukocytes. ${ }^{11}$

The incidence of inflammatory reactions observed in teeth subjected to a combination of pulpal and periodontal irritants is higher than with periodontal disease alone. ${ }^{2}$ Periodontal lesions routinely interfere with nutritional supply of the involved teeth resulting in atrophic and degenerative changes in the pulp. Teeth affected with advanced periodontitis and results specific to periodontally affected teeth have revealed acute and chronic pulpitis, vascular congestion, and pulp necrosis. ${ }^{12}$

Various studies have confirmed the observations of the study, however many other studies have reported differing results. In an extensive study conducted by Mazur and Massler, it was observed that severity or presence of periodontal disease had no effect on the pulp, with similar mild to severe pulp histologic changes being observed in both healthy control and periodontally affected teeth. ${ }^{13}$ Another classic study by Czarnecki and Schilder also did not find any association between the severity of periodontal disease and the presence or absence of pulp pathology. ${ }^{14}$

The literature review suggests conflicting results on the influence of periodontal disease on the dental pulp. With the observations obtained from the present study it is difficult to clearly suggest that the changes observed were limited to diseased teeth and could also be observed in other teeth from the same patient as the test and control teeth are procured from different patients which is the limitation of the study. A controlled study would be the best to identify the influence of periodontal disease on the dental pulp and animal models are ideal for the study.

\section{CONCLUSION}

The present study suggests that teeth with moderate and severe chronic periodontitis exhibit characteristics changes in pulp, but mere presence of periodontal disease does not dictate involvement of pulp and total pulpal disintegration. However, considerations have to be given to the depth of pocket and the severity of periodontal disease to enable the clinician in proper diagnosis, treatment planning and clinical management.

\section{REFERENCES}

1. Novak MJ. Classification of diseases and conditions affecting of periodontium. In: Newman MG, Takei H, Klokkevold PR, Carranza FA (eds), Carranza's clinical periodontology, 10th edition, Saunders, Philadelphia, 2006;100-109.

2. Bender IB, Seltzer $S$. The effect of periodontal disease on the pulp. Oral Surg Oral Med Oral Pathol 1972; 33(3):458-474.

3. Simon JH, Glick DH, Frank AL. The relationship of endodontic-periodontic lesions. J Periodontol 1972; 43(4):202-208.

4. Colyer F. Bacteriological infection in pulps of pyorrhectic teeth. Br Dent J 1924; 45:558.

5. Altman M, Guttuso J, Seidberg BH, Langeland K. Apical root canal anatomy of human maxillary central incisors. Oral Surg Oral Med Oral Pathol 1970;30(5):694-699.

6. Sood M,A study of epidemiological factors influencing periodontal diseases in selected areas of District Ludhiana, Punjab, Indian J Community Med, 2005;30(2):70-71.

7. Russell LH, Kramer IR. Observations of the vascular architecture of the dental pulp. J Dent Res 1956;35:957.

8. Saunders RL. X-ray microscopy of the periodontal and dental pulp vessels in the monkey and in man. Oral Surg Oral Med Oral Pathol 1966;22(4):503-518.

9. Rubach WC, Mitchell DF. Periodontal disease, age, and pulp status. Oral Surg Oral Med Oral Pathol 1965;19:482-493.

10. Seltzer S, Bender IB, Ziontz M. The interrelationship of pulp and periodontal disease. Oral Surg Oral Med Oral Pathol. 1963;16:1474-1490.

11. Ghoddusi J. Ultrastructural changes in feline dental pulp with periodontal disease. Microsc Res Tech 2003; 61(5):423-427.

12. Nemec A, Pavlica Z, Stiblar-Martincic D, Petelin M, Erzen D, Crossley D. Histological evaluation of the pulp in teeth from dogs with naturally occurring periodontal disease. J Vet Dent 2007;4(24):212-223.

13. Mazur B, Massler M. Influence of periodontal disease of the dental pulp. Oral Surg Oral Med Oral Pathol 1964; 17:592-603.

14. Czarnecki RT, Schilder H. A histological evaluation of the human pulp in teeth with varying degrees of periodontal disease. J Endod 1979;5:242-253. 